2013

\title{
A COMPARISON OF PIXEL-BASED VERSUS OBJECT ORIENTED ANALYSIS OF LANDSLIDES USING HISTORICAL REMOTE SENSING DATA
}

Ren N. Keyport

Michigan Technological University

Follow this and additional works at: https://digitalcommons.mtu.edu/etds

Part of the Geology Commons

Copyright 2013 Ren N. Keyport

Recommended Citation

Keyport, Ren N., "A COMPARISON OF PIXEL-BASED VERSUS OBJECT ORIENTED ANALYSIS OF LANDSLIDES USING HISTORICAL REMOTE SENSING DATA", Master's report, Michigan Technological University, 2013.

https://doi.org/10.37099/mtu.dc.etds/704

Follow this and additional works at: https://digitalcommons.mtu.edu/etds

Part of the Geology Commons 


\title{
A COMPARISON OF PIXEL-BASED VERSUS OBJECT ORIENTED ANALYSIS OF LANDSLIDES USING HISTORICAL REMOTE SENSING DATA
}

\author{
By
}

Ren N. Keyport

\begin{abstract}
A REPORT
Submitted in partial fulfillment of the requirements for the degree of MASTER OF SCIENCE

In Geology
\end{abstract}

MICHIGAN TECHNOLOGICAL UNIVERSITY

2013

(C) 2013 Ren N. Keyport 
This report has been approved in partial fulfillment of the requirements for the Degree of MASTER OF SCIENCE in Geology.

Department of Geological and Mining Engineering and Sciences

Report Advisor: Dr. Thomas Oommen

Committee Member: Dr. John S. Gierke

Committee Member: $\quad$ Dr. Michael J. Falkowski

Department Chair: Dr. John S. Gierke 


\section{TABLE OF CONTENTS}

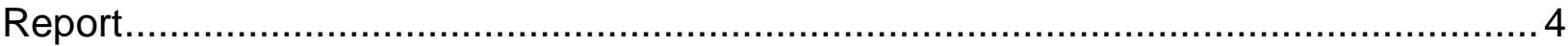

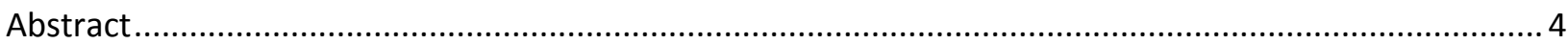

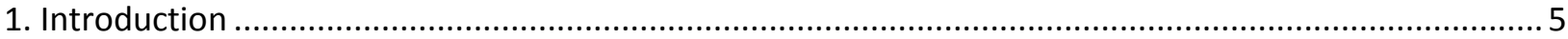

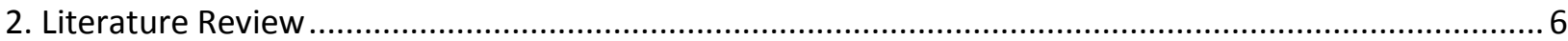

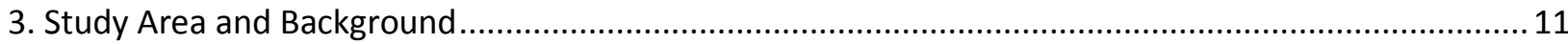

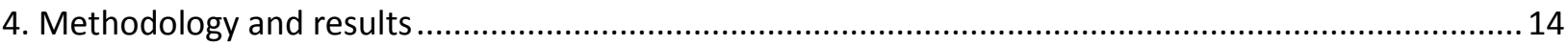

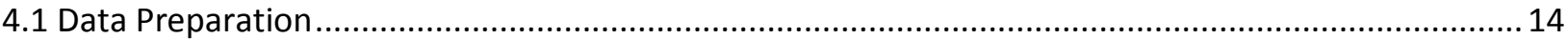

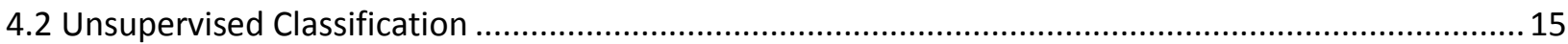

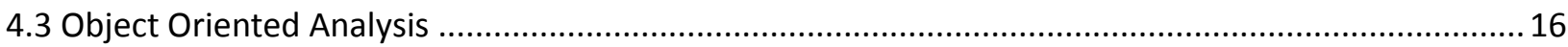

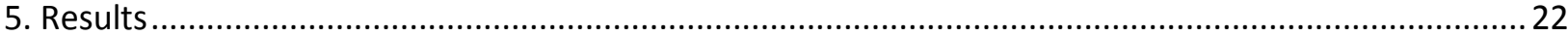

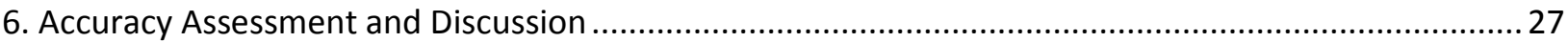

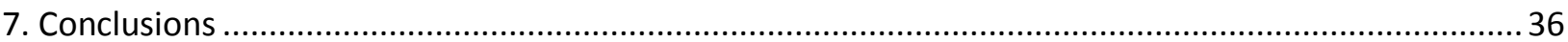

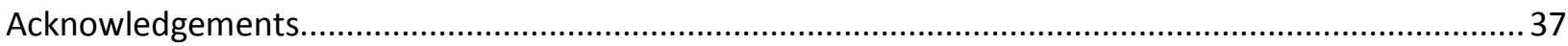

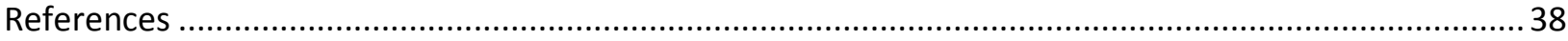

APPENDIX I: Accuracy Assessment Data ........................................................ 41

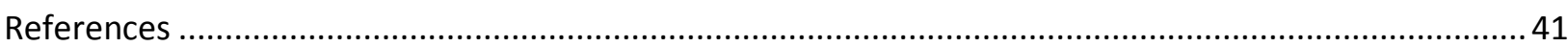

APPENDIX 2: Landslide Inventory and Validation ......................................... 42 


\title{
Report
}

\section{A Comparison of Pixel-Based Versus Object-Oriented Analysis of Landslides Using Historical Remote Sensing Data}

Ren N. Keyport

Department of Geological and Mining Engineering and Sciences, Michigan

Technological University, Houghton, MI, USA

\begin{abstract}
With recent advances in remote sensing processing technology, it has become more feasible to begin analysis of the enormous historic archive of remotely sensed data. This historical data provides valuable information on a wide variety of topics which can influence the lives of millions of people if processed correctly and in a timely manner. One such field of benefit is that of landslide mapping and inventory. This data provides a historical reference to those who live near high risk areas so future disasters may be avoided. In order to properly map landslides remotely, an optimum method must first be determined. Historically, mapping has been attempted using pixel based methods such as unsupervised and supervised classification. These methods are limited by their ability to only characterize an image spectrally based on single pixel values. This creates a result prone to false positives and often without meaningful objects created. Recently, several reliable methods of Object Oriented Analysis (OOA) have been developed which utilize a full range of spectral, spatial, textural, and contextual parameters to delineate regions of interest. A comparison of these two methods on a historical dataset of the landslide affected city of San Juan La Laguna, Guatemala has proven the benefits of OOA methods over those of unsupervised classification. Overall accuracies of $96.5 \%$ and $94.3 \%$ and F-score of $84.3 \%$ and $77.9 \%$ were achieved for OOA and unsupervised classification methods respectively. The greater difference in F-score
\end{abstract}


is a result of the low precision values of unsupervised classification caused by poor false positive removal, the greatest shortcoming of this method.

Keywords Unsupervised Classification; Object Oriented Analysis; Segment Optimization; Landslide mapping

\section{Introduction}

Over the past several years, major advances have been made in the acquisition of high resolution remote sensing data. These advances have led to an influx of data which is collected in hopes of being analyzed, but is often lost in the massive historical archive of aerial and satellite imagery (O'Neil-Dunne 2010). There is great potential to advance our understanding of natural hazards through examination of this data. This examination, if conducted in the most accurate and time effective manner, could save thousands of lives by informing the people of previous incidents which they may face again in the near future.

Traditionally, remote sensing data have been analyzed using pixel based unsupervised and supervised classification approaches which are limited in the image properties which they assess. These methods rely solely on spectral characteristics of the analyzed image which greatly limits the potential for identification of spatially contiguous areas; often resulting in salt and pepper classification with many small regions or single pixels classified as events (Stumpf et al. 2011).

A new solution gaining popularity in the field of image processing is Object Oriented Analysis (OOA). Papers by Stumpf and Kerle. (2011), Martha (2011), Lahousse et al. (2011), Barlow et al. (2006), Chang et al (2012), and Van Den Eeckhaut et al. (2007) all use or analyze OOA methods for the creation of landslide inventories. These methods begin with a spectral classification and then group nearby pixels based on a 
homogeneity scale factor. These objects or segments can then be analyzed further for spatial, textural, contextual, and additional spectral characteristics. This additional processing eliminates false positives which are easily missed by spectral classification alone (O’Neil-Dunne 2010).

It is important to quantify the effectiveness of these methods to determine their usefulness in the processing of historical datasets where available data might be limited. Several papers have been written to assess the effectiveness of OOA versus pixel based methods (Whiteside and Ahmad 2005; Yan et al 2006; Oruc et al 2011; Chang et al 2012); however, none of these assess the effectiveness of landslide detection with limited historical data. Although each case is unique, as is the available data, OOA and pixel based classification methods must be assessed objectively to determine if they can provide accurate and high quality data to the population in need.

One such population is that of San Juan La Laguna, Guatemala. This region experienced a major landslide event as a result of heavy rainfall caused by Hurricane Stan in October 2005. Hundreds of landslides occurred along the slope surrounding the city. Without proper record, it may be soon forgotten and a similar or worse event is certain to happen again. An assessment of the accuracy of unsupervised classification and object oriented analysis of the landslides caused by Hurricane Stan is presented below based on the historical remote sensing data available for this region.

\section{Literature Review}

In order to determine the best methods for analysis, a detailed review of previous works on OOA methods was required. Because of the broad range of topics covered under OOA, the majority of the focus of this review is on the methods for identification and mapping of landslides. Since the goal of this research was not to develop new procedures for OOA of landslides, but instead test what is available, the interpretation of non-landslide based methods would have been beyond the scope of this paper. 
The first step in any OOA procedure is segmentation of the image. The most common and most successful methods for this involve the region growing approach of multiresolution segmentation in the Trimble eCognition software. Multiresolution segmentation begins by examining a pixel's spectral characteristics. Based on the pixel property of interest, it clusters similar pixels with a homogeneity threshold defined by the user. This scale factor will vary from coarse to fine depending on the size of the objects of interest. (Barlow et al 2006, Martha 2011, Lahousse et al. 2011, Lu et al. 2011, Stumpf and Kerle 2011, Van Den Eeckhaut et al. 2007). However, differences arise in the determination of optimal segmentation scale factor which determines object size. These differences range from basic visual interpretation of object scales based on trial and error (Lahousse et al. 2011), to more complicated but automated methods (Martha 2011, Stumpf and Kerle 2011). Additionally, Chang et al. (2012) suggests that edge-based segmentation, an approach which searches an image for object boundaries based on an object's contrast to its neighbors, provides a more objective approach to segmentation than multiresolution segmentation does, but fails to reference the automated methods mentioned above.

Because of the diverse nature of landslides, a single segmentation scale factor does not accurately represent the full spectrum of events and, therefore, multiple scales may be used. All sources found that a finer initial segmentation (smaller scale factor value) with small image objects was preferred for the initial segmentation. This ensures that small landslides will be included in the final inventory. Although this over-segments the larger landslides, these can be re-segmented later using a variety of techniques (Barlow et al 2006, Chang et al. 2012, Martha 2011, Lahousse et al. 2011, Lu et al. 2011, Stumpf and Kerle 2011, Van Den Eeckhaut et al. 2007).

Upon completion of segmentation, classification of landslide candidates then begins. Because of the destructive nature of landslides, they often leave a mark upon the earth which is spectrally different from surrounding features. This provides a good basis for initial classification (Barlow et al 2006, Chang et al. 2012, Martha 2011, Lahousse et al. 
2011, Lu et al. 2011, Stumpf and Kerle 2011). However, there are some limitations in using spectral properties to distinguish landslides. As Barlow et al. (2006) points out, spectral variations are somewhat dependent on vegetation disruption which will not apply to all cases. Landslides which occur in barren landscapes may have little to no spectral variation from their surroundings. Additionally, shadow may be an issue when referencing images in the visual spectrum for both false positive and missed events. Martha (2011) experienced false positive identification with shadows because exposed surfaces revealed mafic landslide material. Barlow et al. (2006) experienced incomplete landslide identification due to shadows concealing spectral changes. An alternative to spectral variation is provided by utilizing LiDAR imagery and morphologic elements to derive candidates. LiDAR can be used for high resolution surface modeling to delineate small changes in topography caused by landslides. This method is highly dependent on available data and proved only moderately successful in its result (Van Den Eekhaut et al. 2007). Ideally, LiDAR images would be used in conjunction with high resolution spectral band images to identify as many landslide candidates as possible (Martha 2011).

Initial classification of landslide candidates is data dependent, but the most successful methods utilize a brightness threshold for the spectral bands. This eliminates all portions of the study area which are spectrally different from the landslide objects, but it includes many false positive objects which are spectrally similar. This threshold for landslide candidates can be determined objectively using K-means clustering (Martha 2011, Stumpf and Kerle 2011).

Since landslide behavior and geometry may vary widely across a study area, it is easier to remove falsely identified landslides with similar characteristics (roads, buildings, farmland, etc.) than it is to define landslides based on a specific rule-set. Once potential landslides have been classified, the removal of false positives must begin. Because of classification overlap with various manmade and natural features, false positives are also difficult issue to confront. OOA improves upon pixel based methods here by 
allowing assessment of spectral, spatial, textural, and contextual characteristics of the image objects while pixel based classification methods are limited to spectral alone (O’Neil-Dunne 2010). Various approaches are again taken here. Most rely on a combination of existing knowledge of the region in conjunction with statistically derived characteristics of the false positives such as slope, object geometry, and spectral properties with respect to location (Barlow et al 2006, Martha 2011, Lahousse et al. 2011, Lu et al. 2011, Van Den Eeckhaut et al. 2007) with Lahousse et al. (2011), Lu et al. (2011), and Van Den Eeckhaut et al. (2007) attempting methods similar to those first demonstrated by Martha (2011). An alternative option is the use of the random forests method which searches for common object characteristics through the use of random decision trees which are tested against a training sample of the study area (Stumpf and Kerle 2011).

With landslides identified and false positives removed, the accuracy of the results must be tested. The results of the OOA based landslide identification studies examined here range from $70-86 \%$ accuracy in landslide detection with overall accuracy up to $97.5 \%$ (Chang et al 2012). Based on the literature, it was determined that the methods of Martha (2011) provided the most automated and objective approach applicable to the available data of this study. Although this approach did not have the highest accuracy values, it was limited by challenges in the data and not the robustness of the methods (Martha 2011).

There has been little work to determine the accuracy of OOA methods compared to the previous standard of pixel based identification and mapping of landslides. Chang et al. (2012) analyzed the difference between OOA and supervised classification and found an incredible difference between the two results. Landslide detection accuracy for OOA was at $84.4 \%$ and $80.5 \%$ for two test sites, while the pixel based supervised classification approaches resulted in detection of only $43.1 \%$ and $76.2 \%$ for the two sites. Both OOA and supervised classification methods were tested using approaches that are significantly different from other leading research in the field. OOA was 
conducted using edge based segmentation rather than multiresolution segmentation, and supervised classification was conducted using the Support Vector Machine (SVM) method instead of Maximum Likelihood (ML) method (Chang et al 2012). These alternatives appear valid, but should be tested against standard practice methods as well.

To gain some idea of what kind of result to expect, literature from other fields on the topic of OOA versus pixel based classification were examined. These were most available in the study of agricultural and environmental issues and also focused on OOA versus supervised classification. Oruc et al. (2011) compared three different supervised classification methods (parallelepiped, minimum distance, and maximum likelihood) to one OOA approach and attained overall accuracies of $64.6 \%, 62.6 \%$, and $66.9 \%$ for pixel based methods and $81.3 \%$ for OOA. This was for general classification of landscape types (settlements, forests, coal waste, etc.) in northern Turkey. Yan et al (2006) and Whiteside and Ahmad (2005) both used ML supervised classification versus OOA and found overall accuracies of $46.5 \%$ and $69.1 \%$ for pixel based respectively, and $83.3 \%$ and $78.0 \%$ for OOA respectively. These clearly show that supervised classification consistently underperforms compared to OOA.

Supervised classification is not always the best method, however. Borghuis et al. (2007) found that unsupervised classification provided better concordance with manually mapped landslides than supervised classification methods. While both supervised and unsupervised classification methods underperformed manual mapping in the Borghuis et al. (2007) study, unsupervised classification demonstrated almost $24 \%$ better concordance with manual methods because it identified a much greater extent of each landslide. Landslide pixel training used in supervised classification limits the search extent to only those pixels within a very specific range while landslides often demonstrate spectral variability within their extent. In addition, the limited verifiable ground truth available for the current study further qualifies unsupervised classification 
as the appropriate analysis method. Based on these findings, a comparison of OOA and unsupervised classification methods warrants investigation.

\section{Study Area and Background}

San Juan La Laguna $\left(14.695^{\circ} \mathrm{N}, 91.287^{\circ} \mathrm{W}\right)$ is a small community of approximately 10,000 residents located on the shores of Lake Atitlan in south central Guatemala. Lake Atitlan is a caldera lake which is located in the Atitlan III caldera. The most recent major eruption in the Atitlan III caldera occurred 84,000 years B.P. and resulted in the creation of Lake Atitlan. Additionally, three stratovolcanoes have formed on the southern edge of the lake, San Pedro being the closest of the three to San Juan (Newhall et al. 1987). Figure 1 shows the location of San Juan in reference to the country and Lake Atitlan. 

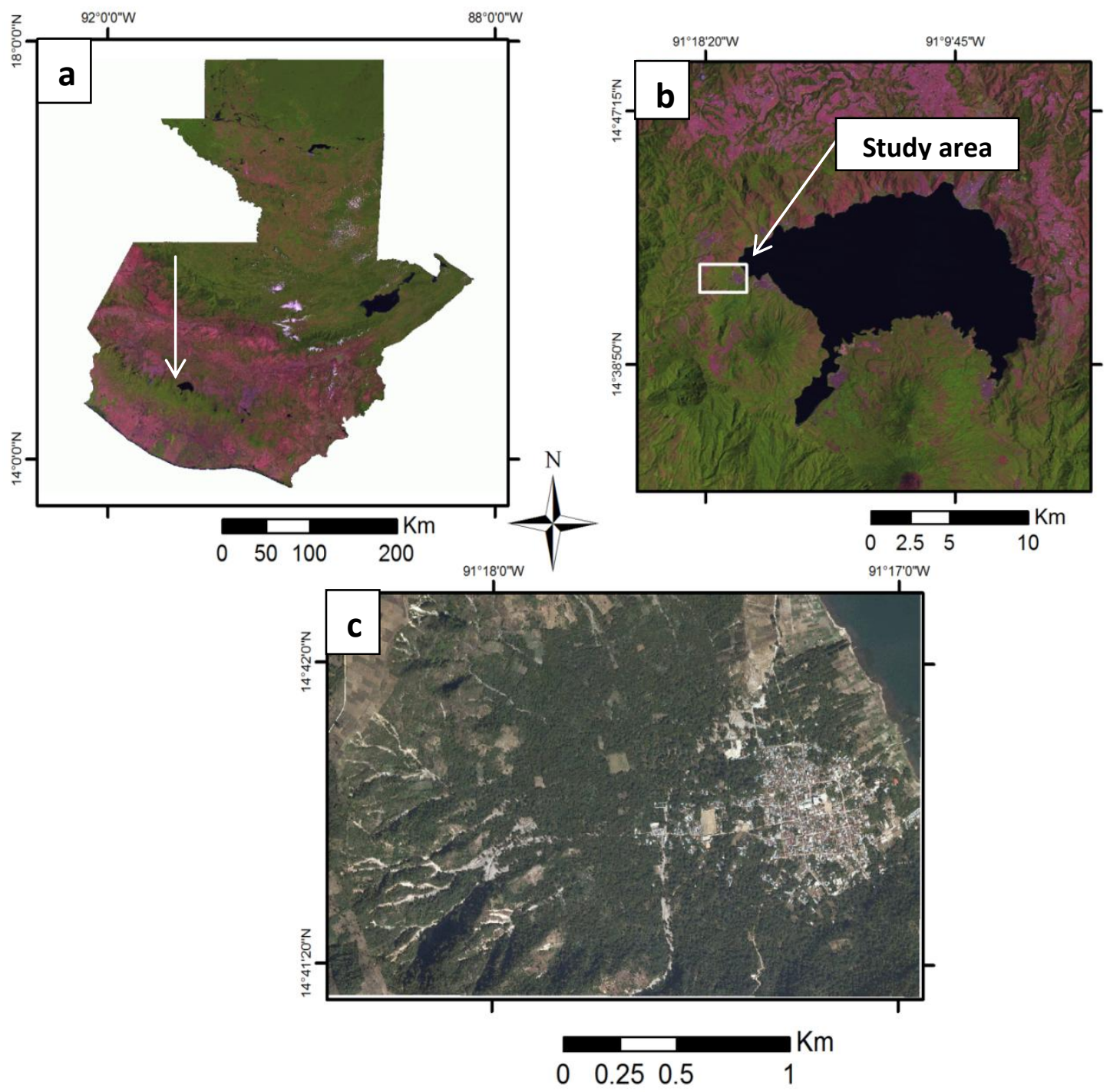

Figure 1: Location of San Juan La Laguna study area on western shore of Lake Atitlan, Guatemala. a. Map of Guatemala showing the location (white arrow) of Lake Atitlan and general location of San Juan and survey area. b. Lake Atitlan and location and extent of study area on the north western shore of the lake. c. Actual study area and reference image used for processing.

This combination of volcanic events has produced a steep sided and often unstable crater rim. Beneath this rim there are several villages which are particularly susceptible to landslides and lahars. At San Juan, slopes reach angles of 80 degrees and only a thin layer of clay and organic soil is present. This thin layer of approximately 1 meter of soil 
creates a very unstable setting which, if subjected to heavy rainfall, is likely to collapse (Luna 2007).

In October 2005, Hurricane Stan provided the heavy rainfall needed to release significant sections of the crater rim. Thousands of landslides occurred along the steep crater walls culminating in the destruction of communities and the loss of hundreds of lives in nearby Panabaj. At San Juan, the impact was fortunately much less. No lives were lost, but significant damage affected the fragile state of the predominantly subsistence farming community. Despite the great toll this event took on the local population, very little scientific effort was put into delimiting and mapping the landslides which occurred during hurricane Stan (Luna 2007). A simple inventory was collected of landslide initiation points by the Instituto Nacional de Sismologia, Vulcanologia, Meterologia, e Hidrologia (INSIVUMEH) and this has proved useful to this study; however it only provides very limited information about the site. Fortunately, high resolution orthophotos were taken shortly after the event in early 2006 . These multispectral (red, green, and blue band) photos at $0.5 \mathrm{~m}$. spatial resolution provide a fairly detailed look at the region and are used in this study to create a proper landslide inventory of the San Juan region. Additionally, 10 meter contours were available with which an approximate Digital Elevation Model (DEM) could be created to represent the topographic properties of the region.

This dataset provided a good basis to test OOA and unsupervised classification methods for remote landslide detection, and to determine the limitations of each. The extent of the region was chosen to represent a broad range of physical features including developed land such as roads, buildings, and farmland, as well as natural features such as rivers and drainage channels, lakeshore, and a variety of other terrain features. This was done intentionally to test the robustness of each method. The two methods selected for examination in this paper are unsupervised classification, and OOA as per methods outlined in Martha (2011). Unsupervised classification was conducted in Erdas Imagine 2011 and OOA was conducted in Trimble's eCognition Developer software. 


\section{Methodology}

\subsection{Data Preparation}

The selection of a study area was based upon available data and familiarity with the region. Since this study was to be conducted on a historical dataset, field work would not be possible for reference, and some form of familiarity with the region was important. Collaboration with a returning Peace Corps volunteer who was stationed in the town of San Juan proved very useful for data collection and site reference.

The extent of the study region was selected to represent the variety of features one might find in a much larger survey area, but was limited due to data processing capabilities and could not include the entire extent of the affected region. Instead a $4.53 \mathrm{~km}^{\wedge} 2$ region was chosen which contained as many of the false positive landslide cases identified in Martha (2011) as possible to test the robustness of OOA and unsupervised classification methods.

A DEM of the region was generated from 10 meter topographic lines. Although the resolution is far lower than that of the reference image, it was the best available dataset and represents one of the limitations of working in remote parts of developing countries. This DEM was then resampled to 0.5 meter resolution in ArcMap 10 and from it slope, flow accumulation, and curvature layers were developed. Data processing can be followed in the process flow chart presented in Figure 2. 


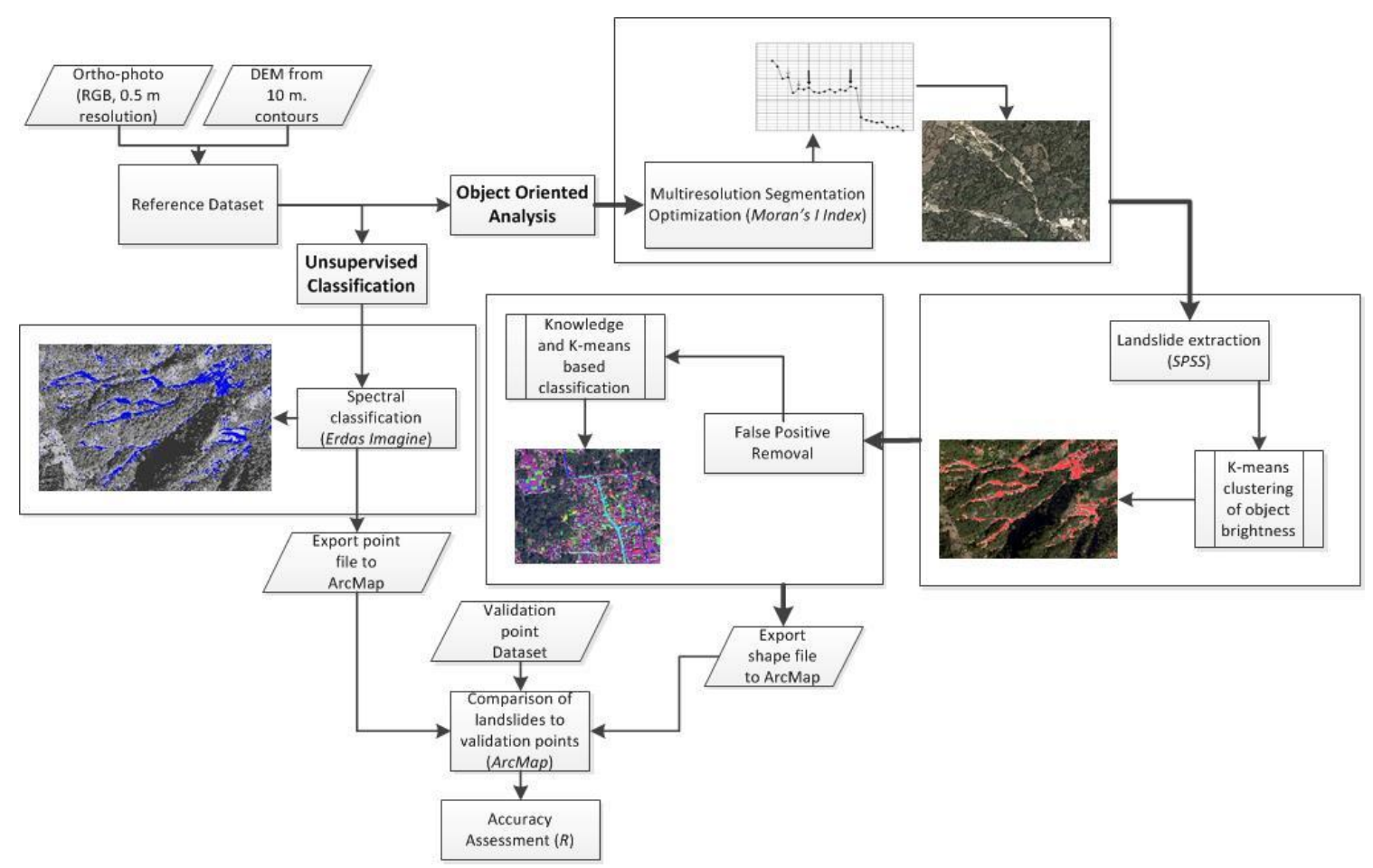

Figure 2: Process flow chart of methods used in OOA and unsupervised classification. Key processing steps are grouped with examples of data result.

\subsection{Unsupervised Classification}

Pixel based classification methods have been widely used throughout the fields of geology, agriculture, military intelligence, and many more (Borghuis et al. 2007). These methods vary in complexity depending on the data available for classification. Since the only high resolution data available for this region was 0.5 meter orthophotos in visible spectrum, the processing methods were limited to basic delineation by spectral properties.

The unsupervised classification was performed using the ISODATA algorithm in Erdas Imagine 2011. Parameter selection is very limited in unsupervised classification with the primary variable being number of classes. Rather than using visual means to determine the optimum classification, an objective approach was developed. The reference image was classified in grey scale using a range of class values from 4-14. In each of these cases, the brightest class best represented the landslide cases in the 
study area. This is a result of the felsic clays created by ash falls from the nearby volcanoes. The fresh landslides generally appear highly contrasted to the surrounding vegetation. Each of these 11 variably classified images was then compared to a reference dataset which will be later discussed in the validation and accuracy assessment section of this report.

\subsection{Object Oriented Analysis}

OOA is a much more complex method of image classification which allows far greater flexibility in the parameterization of inputs. This creates the potential for many different approaches to landslide identification, and determining which method is best may prove challenging. A variety of approaches were examined (Martha 2011; Stumpf and Kurle 2011; Lu et al. 2011; Lahousse et al. 2011; Ruedi et al. 2008). For this study, the procedure outlined in Martha (2011) was found to be the most fitting to the available dataset. This approach uses mostly automated methods to delineate landslides and can easily be translated for use in the San Juan region.

There is some variation in the data between Martha (2011) and this research, and this has required a bit of interpretation and refinement of the methods. The biggest difference is that the reference image used by Martha (2011) was a multispectral image in the red, green, and near-infrared bands; whereas, the image used for this study is in red, green, and blue spectral bands. The second difference is in the spatial resolution of the images. The images used in Martha (2011) had a resolution of 5.8 meters and the resolution of this image is $0.5 \mathrm{~m}$. providing much more detail of the much smaller region. This higher resolution resulted in processing limitations because of the large file size, thus reducing the extent of the region available for processing.

OOA began with the determination of an optimum scale factor with which to base multiresolution segmentation of the image upon. This was done using the objective 
function from Espindola et al. (2006) and the methods outlined by Martha (2011). In eCognition, scale factors for multiresolution segmentation were tested from $10-80$. This range was selected visually to represent a full range of over-segmentation to undersegmentation. Not all scale factors were tested, from 10-30 every fifth was tested and from 30-80 every second scale factor was tested. At each scale factor, the brightness, standard deviation of each layer, and pixel area for each segment were exported as shape files for analysis in ArcGIS. From here, Moran's I index, a measure of spatial autocorrelation, was calculated at each scale factor based on the variance $\boldsymbol{v}$ of the objects in the brightness layer (Martha 2011, Espindola et al 2006).

$$
v=\frac{\sum_{i=1}^{n} a_{i} * v_{i}}{\sum_{i=1}^{n} a_{i}}
$$

Where $\boldsymbol{a}_{\boldsymbol{i}}$ is the area of each object and $\boldsymbol{v}_{\boldsymbol{i}}$ is the intra-segment variance of object $\boldsymbol{i}$. Moran's I index $(I)$ follows the equation:

$$
I=\frac{n}{S_{0}} * \frac{\sum_{i=1}^{n} \sum_{j=1}^{n} w_{i, j} z_{i} z_{j}}{\sum_{i=1}^{n} z_{i}^{2}}
$$

Where $\boldsymbol{n}$ is the total number of objects, $\boldsymbol{w}_{\boldsymbol{i}, \boldsymbol{j}}$ is the spatial weight between two objects $\boldsymbol{i}$ and $\boldsymbol{j}, \boldsymbol{z}_{\boldsymbol{i}}$ is the deviation from mean of the brightness of each object, and $\boldsymbol{S}_{\mathbf{0}}$ is the sum of all spatial weights:

$$
S_{0}=\sum_{i=1}^{n} \sum_{j=1}^{n} w_{i}, j
$$

The area and standard deviation of each segment at each scale factor were then used to find the weighted variance and the normalized objective function according to the same methods as Martha (2011) expressed as:

$$
F(v, I)=F(v)+F(I)
$$

and the functions $\boldsymbol{F}(\boldsymbol{v})$ and $\boldsymbol{F}(\boldsymbol{I})$ are normalized according to: 


$$
F(x)=\frac{X_{\max }-X}{X_{\max }-X_{\min }}
$$

A plot of scale factor and objective functions was used along with a plateau objective function by Martha (2011):

$$
F(p)=F(v, I)_{\max }-\sigma
$$

Where $\boldsymbol{\sigma}$ is the standard deviation of the objective function and $\boldsymbol{F}(\boldsymbol{v}, \boldsymbol{I})_{\max }$ is its maximum value. This aided in the determination of the optimum scale factor by delimiting the first standard deviation from the maximum Moran's index (I) value. According to Martha (2011) Moran's Index is "an indicator of intrasegment homogeneity and intersegment heterogeneity". Objective function peaks were then selected above this plateau in a similar manner to that in Martha (2011). Since only two peaks fell above the plateau objective function, two peaks were selected below the plateau as well for further testing. Figure 3 compares the plateau objective function plots of Martha (2011) to the one derived from this study. This is discussed further in the results section below. 

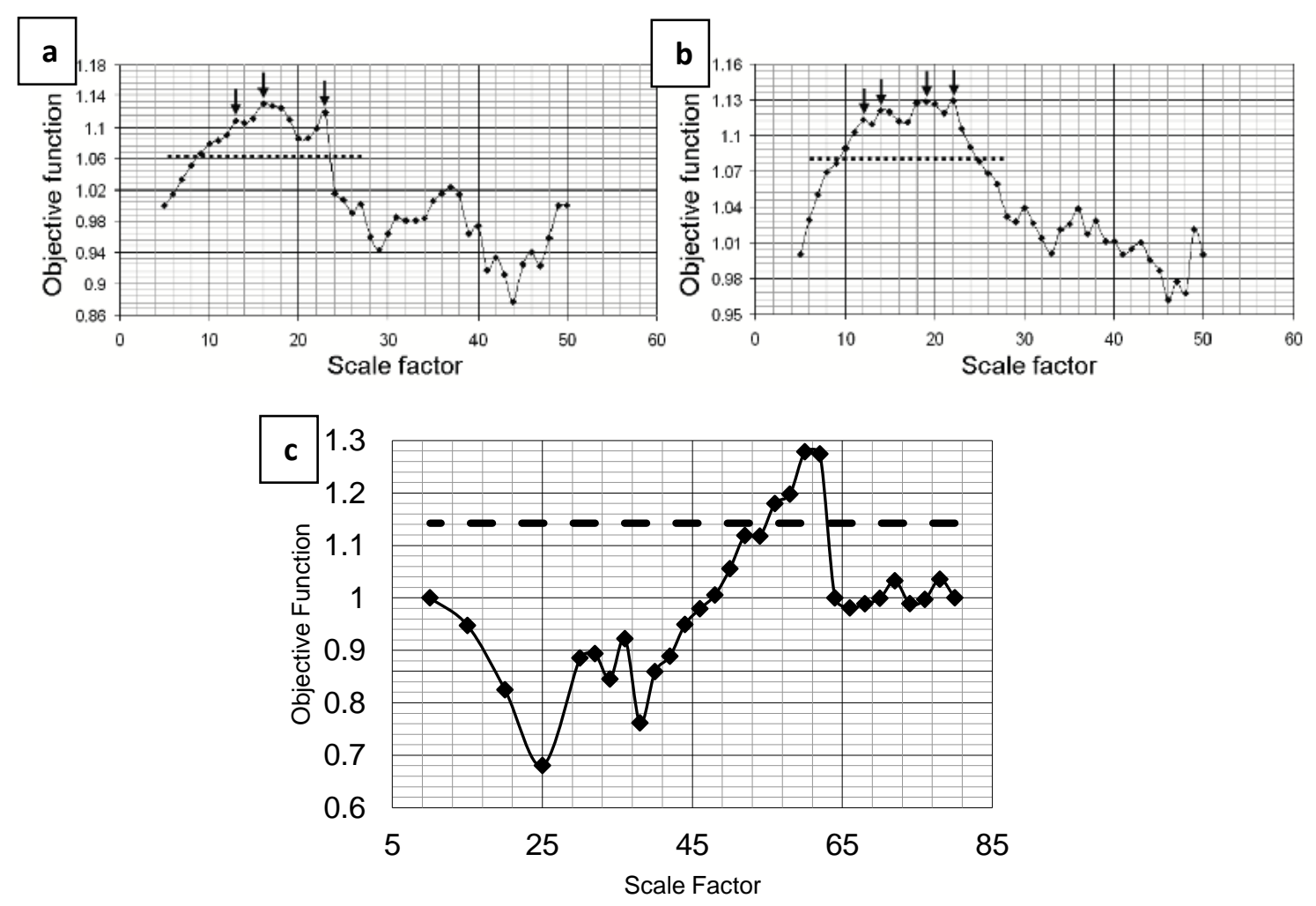

Figure 3: Objective functions from Martha (2011) and this study. a. \& b. show the objective functions from Martha (2011) two study areas, c. shows the objective function from this study for comparison.

Extraction of landslide candidates followed. Multiple scale factors were picked from the objective function plot to be tested. The first peaks above the plateau objective function were picked in accordance with the methods outlined in Martha (2011). The smallest should outline all small landslides and also accurately portray boundaries of larger landslides. The second, larger scale factor was also selected to test the robustness of these methods because it visually appeared to satisfy the same requirements of the finer scale factor, and appeared as the largest peak on the somewhat ambiguous result. The two peaks below the plateau objective function were also selected to test robustness of this approach. 


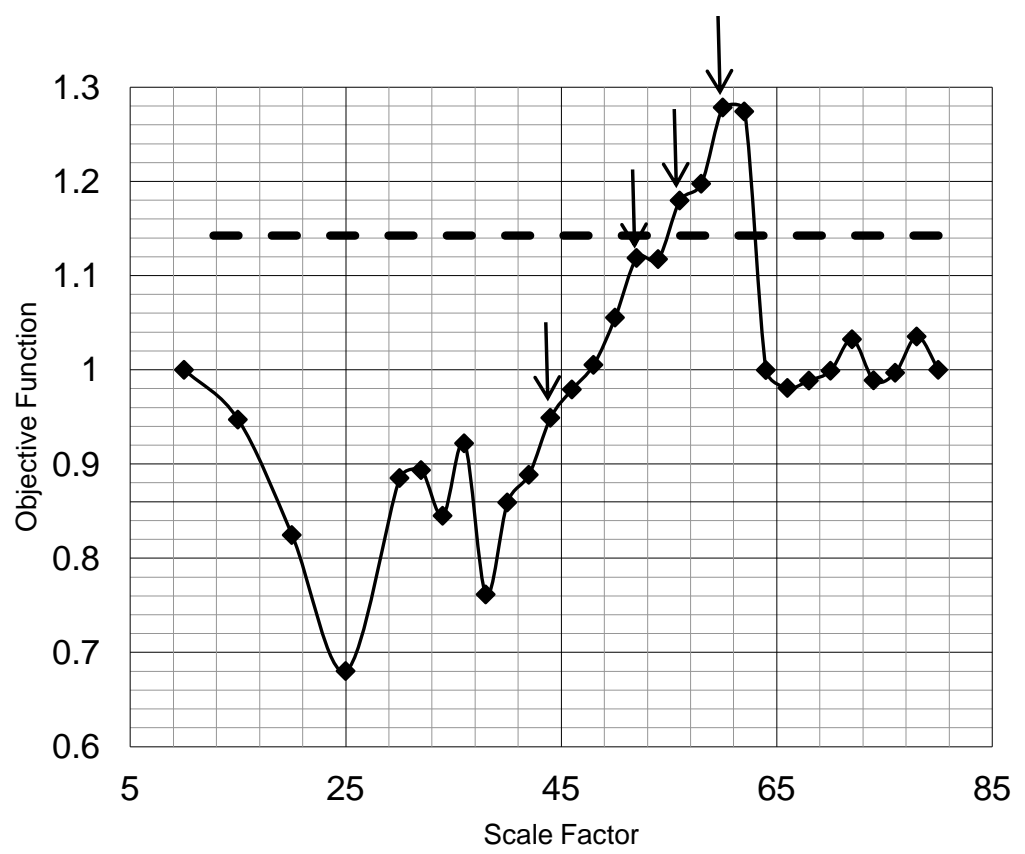

Figure 4: Plot of scale factors vs. objective function for the determination of optimum scale factor. Arrows indicate tested scale factors with bold arrows being processed completely. Dashed line indicates plateau objective function, above which all valid classification criteria should exist.

Landslide candidates were then extracted using a brightness threshold derived by two step k-means clustering in IBM's SPSS predictive analytics software. Brightness values were exported from eCognition based on the average of the three spectral bands for each image object. Using these values, two step K-means clustering began by automatically determining the ideal number of cluster centers for the K-means clustering algorithm. This process was followed exactly as in Martha (2011); however, it did not provide a reasonable result. The calculated cluster centers were skewed too high and too low for successful landslide detection and it was assumed that this was because too few cluster centers were being used. The number of desired clusters was instead determined based on a visual interpretation of the data by using a rough estimate of a brightness threshold. Assumed landslide objects were tested for their brightness values and a minimum brightness threshold was estimated based on this. With this value in mind, incrementing cluster center values were tested in K-means 
clustering until a value similar to the estimate was derived. The calculated brightness value close to the visually approximated value was then tested in eCognition on the survey area. The application of this threshold eliminated all regions spectrally darker than the landslide regions.

Elimination of false positives was necessary since only a brightness threshold was used to select candidates. Other features such as farmland, roads, buildings, beaches, and rivers were also detected with the single threshold. The elimination of these false positives was conducted with the use of existing knowledge of the region, and spatial characteristics of the objects derived by K-means clustering. It is much easier to identify spatially consistent objects, such as those mentioned above, than it is to define the characteristics of erratic and inconsistent landslide objects. For this reason, false positive elimination is preferred over in depth landslide characterization (Martha 2011). Table 1 outlines each parameter for false positive elimination.

Table 1: Parameter selection for OOA of landslide cases at scale 44. Object character types remained the same, but values varied for other scale factors tested. The first target was the selection of landslide candidates. The following five eliminated false positives based on object character derived using the listed method.

\begin{tabular}{|l|l|l|l|}
\hline Target & Property & Object Character & Method \\
\hline Landslides & Spectral (RGB) & Mean Brightness $>138$ & K-means clustering \\
\hline Rooftops & Object Geometry & Rectangular fit $>.87$ & Existing Knowledge \\
\hline Barren Lands & Spectral (Blue) & Mean difference $>100$ & K-means clustering \\
\hline Roads & Object Geometry & Compactness $>4.5$ & K-means clustering \\
\hline & Object Geometry & Length/width $>6$ & K-means clustering \\
\hline Developed land & Slope & Mean Slope $<9$ degrees & Existing Knowledge \\
\hline
\end{tabular}

Object geometry was critical in the identification of false positives. Rectangular fit is a measure of the closeness of fit to a perfect square (value 1). Since buildings are often rectangular in shape, this is a useful parameter for their identification. The high reflectivity of their barren lands isolates them from their neighbors. A mean difference from darkest neighbor threshold was used in eliminating some barren land objects. 
Compactness describes the shapeliness of an object, and is therefore useful in defining road networks which have a high compactness value. The length to width ratio of an image object is also useful in defining road objects which have are generally much longer than they are wide. Classification was also based partially on existing knowledge of the region. Existing knowledge results are based on the use of the feature extraction tool in eCognition.

\section{Results}

Unsupervised classification was conducted using 11 different classification scales. These scales divided the three band image into 4-14 different classes based on the brightness value of the combined RGB layers. The range was selected visually based on over- and under-classification of the image. Although this is an empirical method, it proved reliable in the data trend of the processed result. Figure 5 shows the 4 and 14 class unsupervised classification results to demonstrate over and under-classification. 

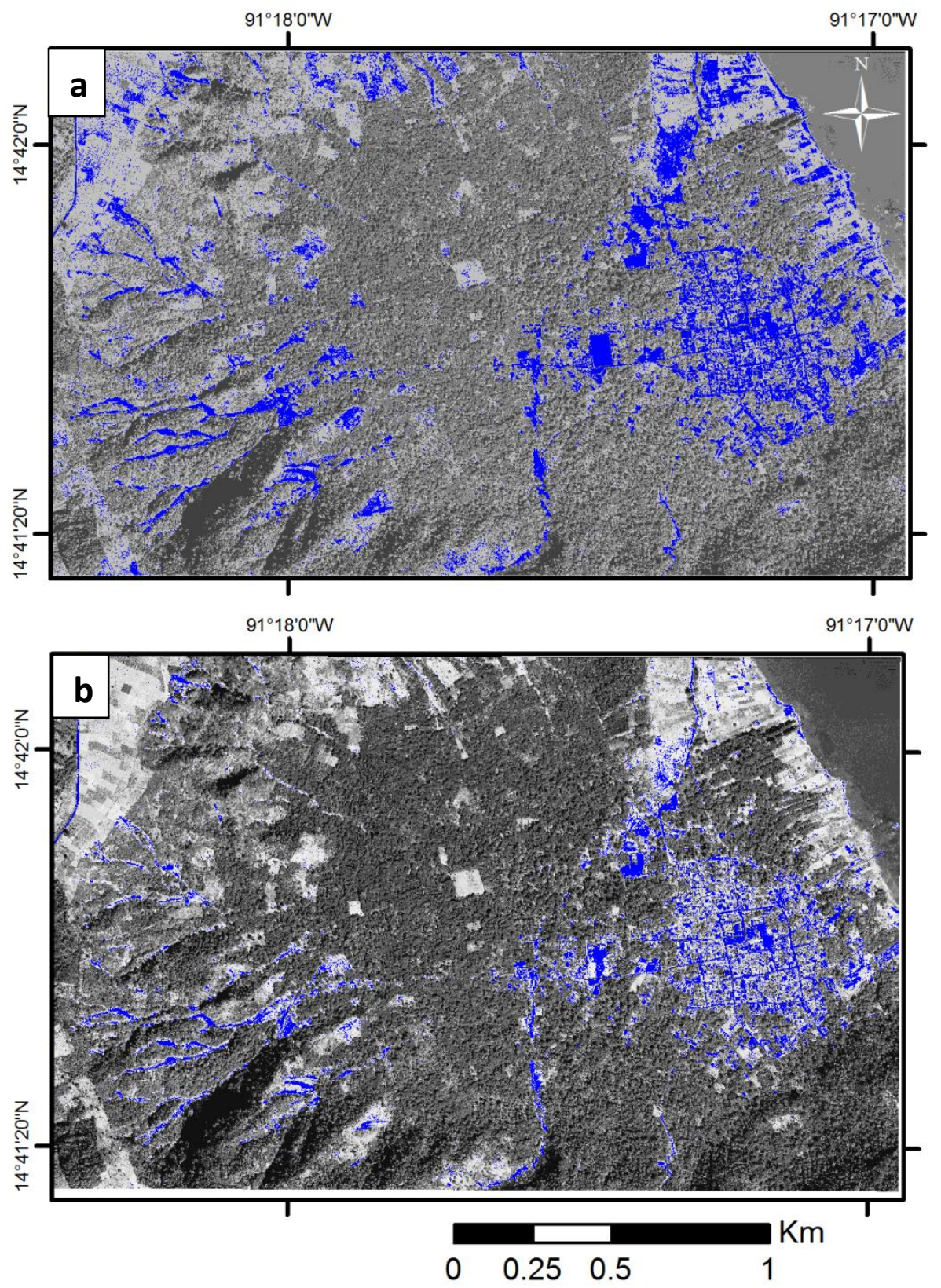

Figure 5 Under-classified and over-classified results of landslide identification. Blue pixels identify potential landslide candidates. a. Under-classified result with 4 pixel classes determined to be under-classified because pixel extent exceeds boundaries of landslide regions. b. Over-classified result with 14 pixel classes is clearly missing significant portions of landslide extent near landslide boundaries. 
Upon completion of unsupervised classification, OOA began. Selection of an optimum scale factor for the OOA processing flow required the analysis of the objective functions outlined in equations 4.1-4.6. According to Martha (2011), the optimum scale factor should be selected as the first peak (smallest scale factor) above the plateau objective function. To test this hypothesis, the two peaks above the objective function were selected as well as two peaks below at smaller segmentation scale factors. Figure 4 shows the objective function results according to the methods defined in Martha (2011). Figure 6 compares a small region at two scale factors to represent the effect of a range of segmentation values on object size.

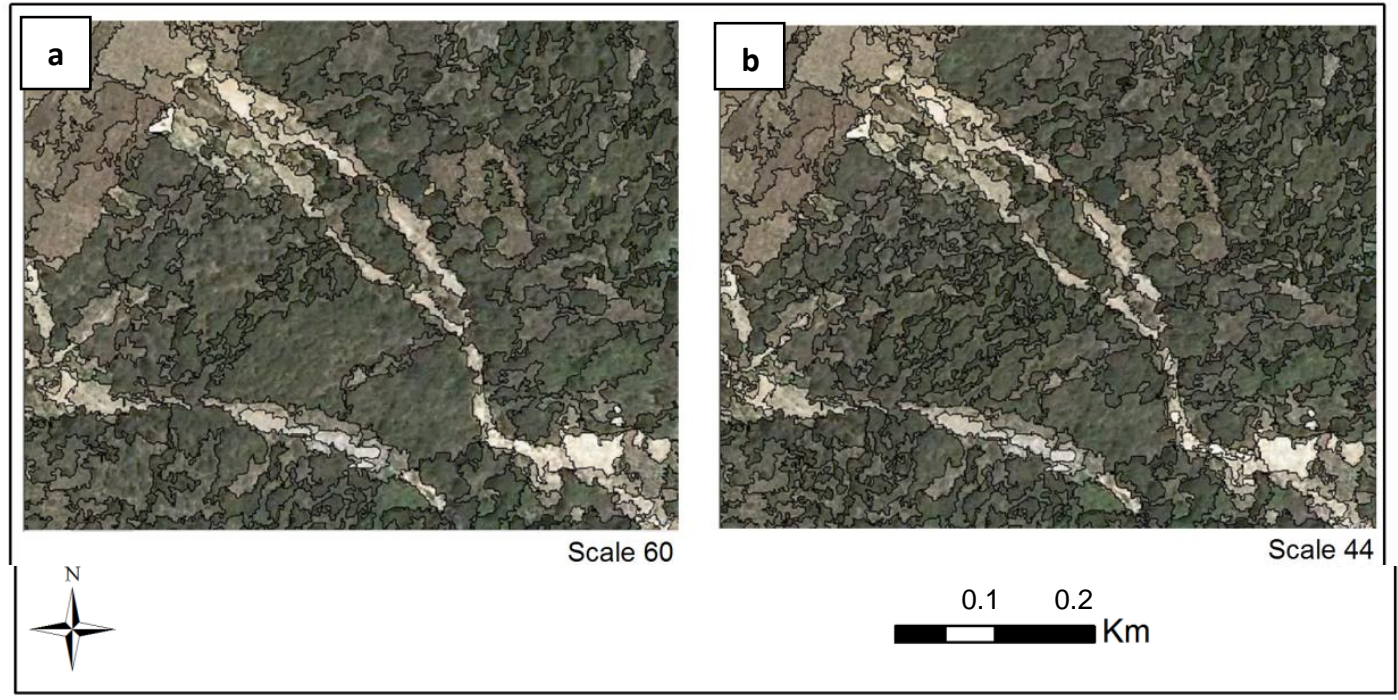

Figure 6: Segmentation of the same landslide at two scale factors showing the variation in object extent based on multiresolution scale factor.

With a range of segmentation values selected for testing, landslide detection could begin. Martha (2011) provided a two-step k-means clustering approach for initial landslide detection using image brightness. This step is critical for eliminating large regions of the image which do not belong to the landslide class based on their spectral characteristics such as dense vegetation, water, and some agricultural lands. This process, when conducted using SPSS Statistics predictive analytics software, resulted in the just two clusters of brightness, neither of which accurately represented the landslide regions. The cluster center for the brightest region had a brightness value of 
165 which, when tested, eliminated a large portion of the landslide objects with lower reflectance. This is likely due to the large developed area of the image constituting the town of San Juan. Here many buildings, roads, and parking areas have very high brightness values. To overcome this, an estimate was made of an approximate brightness value which would allow all actual landslides to be included in the landslide candidate class. This brightness threshold of 140 was determined by examining brightness values of assumed landslide objects with eCognition's feature extraction tool. Utilizing this value, K-means clustering was conducted using increasing numbers of centers until a value close to 140 was attained. This occurred at 4 cluster centers, which is the same number of centers used in Martha (2011). A brightness value of 138 was calculated as the second brightest cluster center delimiting the lower boundary of landslide brightness. This value was used as the initial threshold for potential landslides. All image objects with a mean brightness of less than 138 were eliminated from the candidate pool.

Brightness thresholding creates a rough estimate of potential landslides within the study area. It also identifies many landforms with similar spectral characteristics such as roads, farmland, barren land, and buildings. These falsely identified landslides are referred to as false positives. False positives identified in the brightness based classification were almost exclusively the result of manmade features. The main false positive region being that of the town of San Juan with its many high brightness features. Fortunately, these features have some similar geometric and contextual properties which are different from most landslides. Rectangular fit is used in the identification of rooftops, parking lots, and roads. Compactness and length/width ratio are useful in the identification of roads and road networks which might be connected into single objects. Brightness characteristics vary too. The mean difference of an object to its neighbors was also useful in the elimination of developed lands. Two step K-means clustering was used in the derivation of rectangular fit, compactness, length/width, and mean difference false positive elimination thresholds. The remaining 
false positives are comprised mostly of low lying farmlands and open spaces concentrated near and within the city and do not exist on the steep slopes near San Juan which are most susceptible to landslides. Because of this, slope was selected for the final false positive elimination parameter. Figure 7 displays all landslide candidates as colored objects with each false positive colored differently based on its respective parameter of elimination.

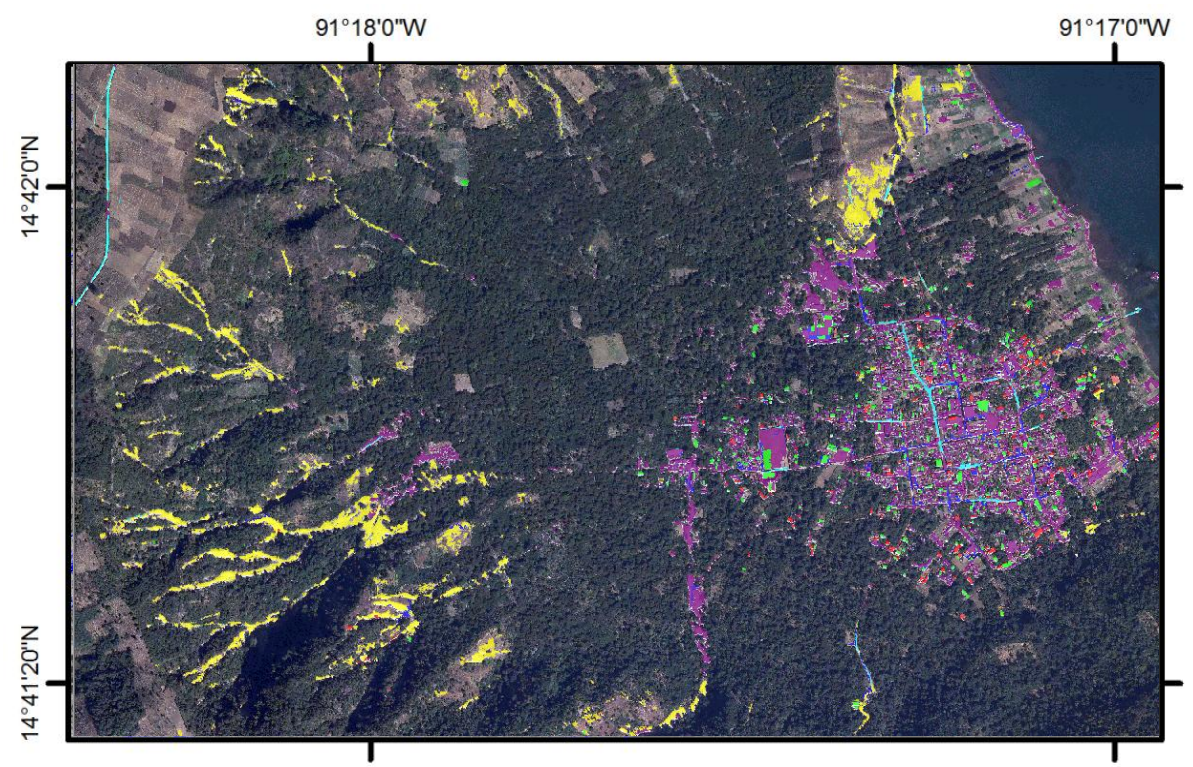

\section{Legend}
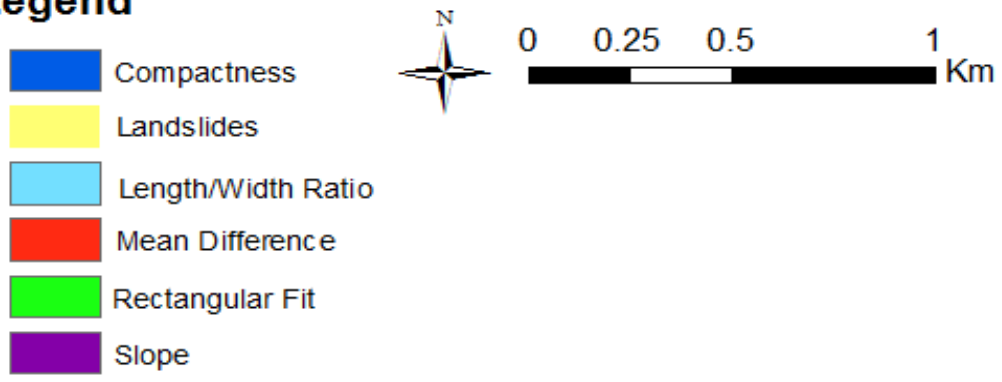

Figure 7: OOA result with segmentation scale factor 44 . All colored objects indicate regions identified as potential landslides based on initial brightness threshold. False positives were then eliminated with thresholds described in Table 1 and are colored based on this parameter.

It is important to note that this process did not provide perfect identification of all landslide candidates, or elimination of all false positives. Ambiguity in the dataset and variability of the spectral characteristics of the landslides made some identification by 
OOA and unsupervised classification impossible. In particular, shadowed regions containing landslides were not identified in either OOA or unsupervised classification because of the vast difference in brightness value caused by the shadows. Figure 8 shows three landslides hidden by a shadow which resulted in missed identification.

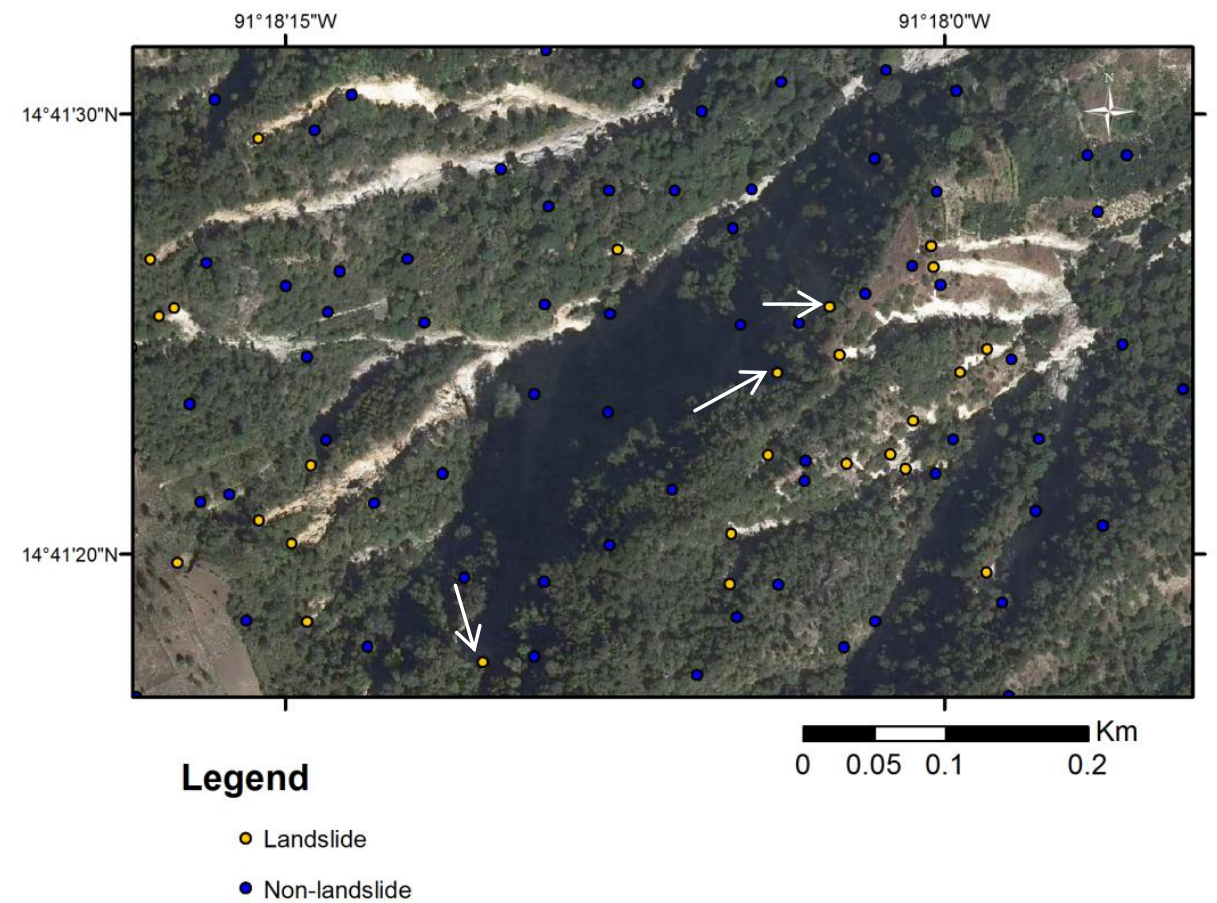

Figure 8: Landslide events missed because of concealment by shadows are denoted by white arrows. These events have no spectral variation from the shadow region and could not be identified using only a single spectral image.

\section{Accuracy Assessment and Discussion}

The development of an assessment of the accuracy of unsupervised versus OOA methods of landslide detection required the creation of a validation dataset with which to compare the results of each method. This consisted of the ground truth dataset provided by INSIVUMEH and a randomly generated group of 900 data points associated with non-landslide cases. 
The ground truth dataset was in the form of GPS located initiation points for every landslide which occurred after hurricane Stan in October 2005. This meant that each landslide only had one verifiable point. This limits the validation potential, but again represents the challenges of working with historical data from a remote region of a developing nation. To add to the challenges of this dataset, the accuracy of each point was low enough to place some points outside of the actual landslide boundary. This is likely due to the incredibly steep terrain and inaccuracy of data collection methods. The few reference points that were visually outside of the boundaries of the landslides were adjusted to be visually within the boundary of the landslide which they appeared to be referencing. In all cases it was easily distinguished which area of the image they were meant to represent. A total of 115 landslide initiation points were identified within the study area, each composed of a single pixel. Figure 9 shows a small portion of the study area with corrected initiation points. For the complete landslide inventory see Appendix 2. 


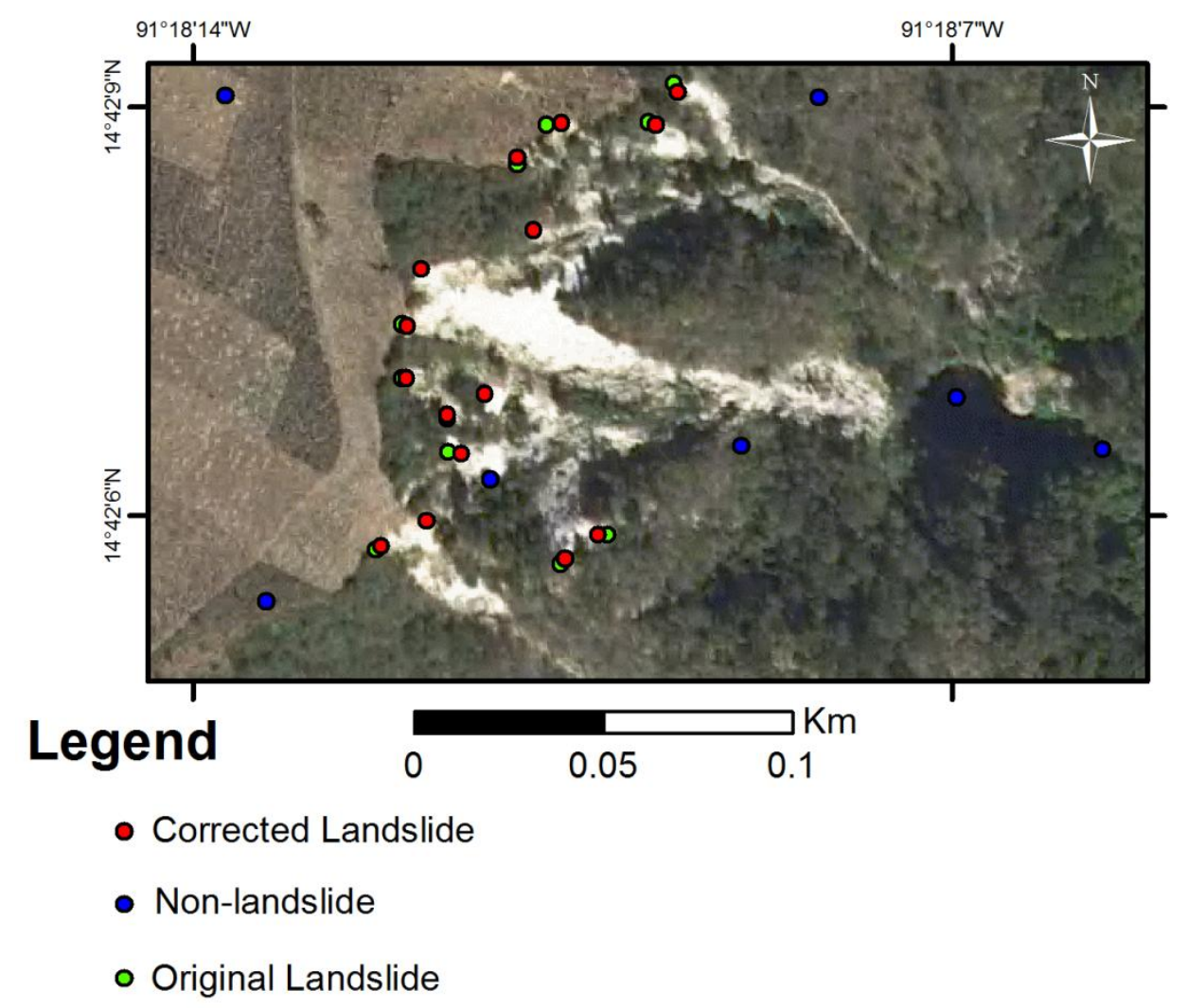

Figure 9: Zoomed map indicating landslide initiation points that were corrected due to inaccuracy in data collection. Green indicates the original location of the initiation point and red is the corrected location which falls definitively within the indicated landslide.

The non-landslide validation dataset was created by the selection of 900 random points across the image. Those points which were adjacent to landslide initiation points and within the apparent landslide boundary, as well as those which landed ambiguously within potential landslide boundaries, were removed from the dataset to avoid false classification. Since the full extent of the landslides was not confirmable with ground truth, these boundaries had to be inferred based on spectral continuity of the region identified by the initiation points. This method proved successful for all but one data point which rested in a drainage channel beneath several landslide initiation points. The exposed channel has the same spectral characteristics as the associated landslides and it is impossible to tell from the reference image where the landslides end and the 
more permanent channel begins. Because of this ambiguity, this point was removed as well. This resulted in a data set of 894 non-landslide reference pixels.

These two validation datasets were combined and a binary reference was assigned with value 0 for landslide and 1 for non-landslide pixels. Extraction of values to these data was then conducted in ArcMap for each of the 11 unsupervised classification and the four OOA shape files. Each produced a file of 1009 true positive, true negative, false positive and false negative data points to calculate the accuracy of each method.

From these data, an accuracy assessment was conducted in R which calculated overall accuracy, precision, recall, and F-score of each classified dataset based on the equations outlined in Appendix 1. Overall accuracy is the arithmetic mean of the correctly identified landslide and non-landslide regions, or how many points were correctly picked overall. Precision of landslides is the number of landslides that were picked out of all the points identified as landslides. High values indicating more landslides were identified as landslides than non-landslides were. Recall of landslides is the percentage of all landslide events that were identified. A high recall value indicates that most of the events were mapped. F-score is the harmonic mean of the landslide and non-landslide validation datasets and is important because the number of nonlandslide cases far outnumbers the number of landslide cases. By using precision and recall, no extra weight is given to the higher number of non-landslide events (Oommen et al. 2010). Rather than selecting the single best unsupervised classification, all 11 results were included in Figure 10 and Figure 11 to show the variability, advantages and disadvantages of each. 

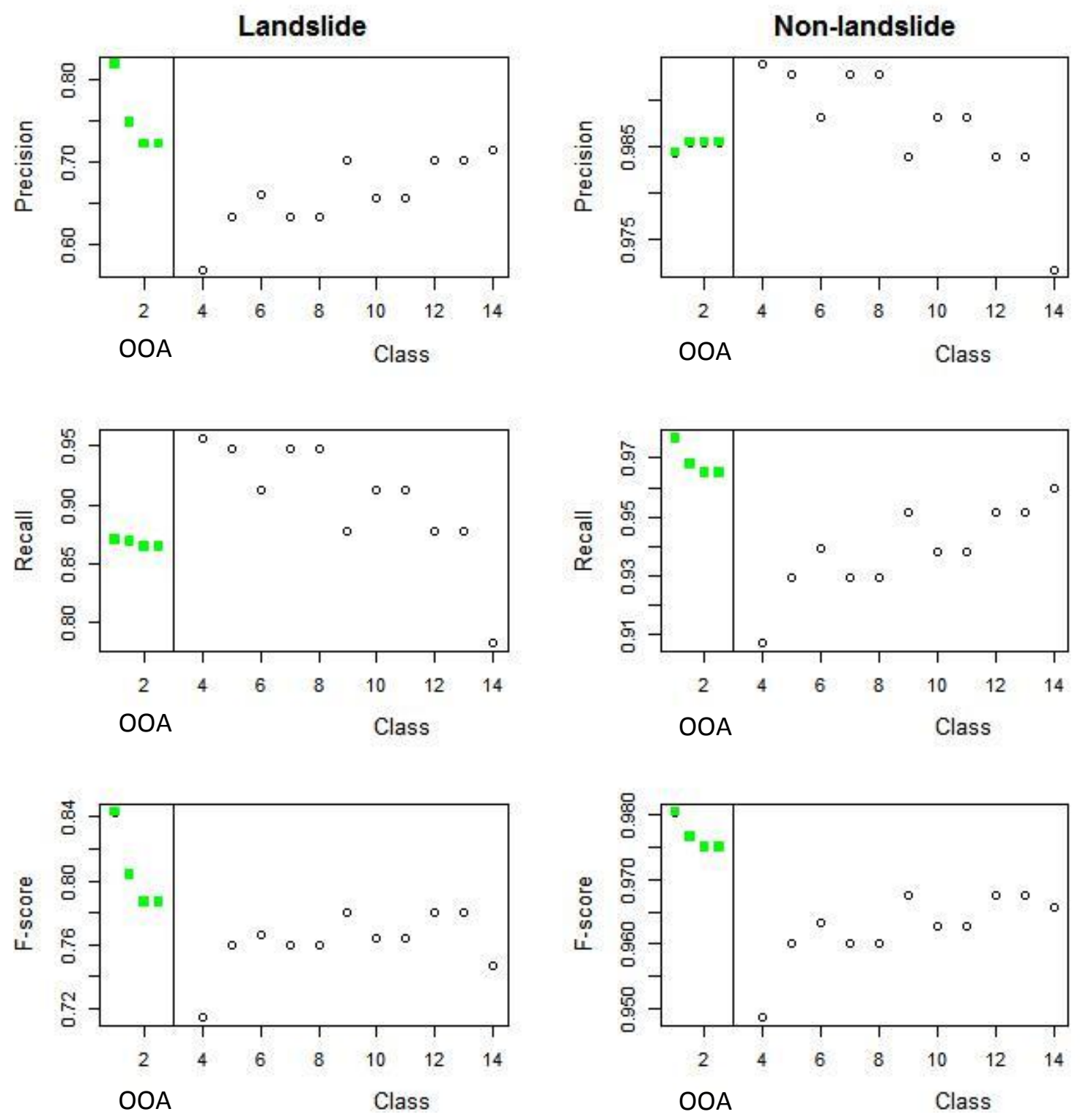

Figure 10: Precision, recall, and f-score plots for comparison of landslide and nonlandslide identification results. OOA scale factors are plotted in green squares incrementally from 44-60. Class 4-14 indicate unsupervised classification levels as indicated by number. All equations and values may be referenced in Appendix 1. 


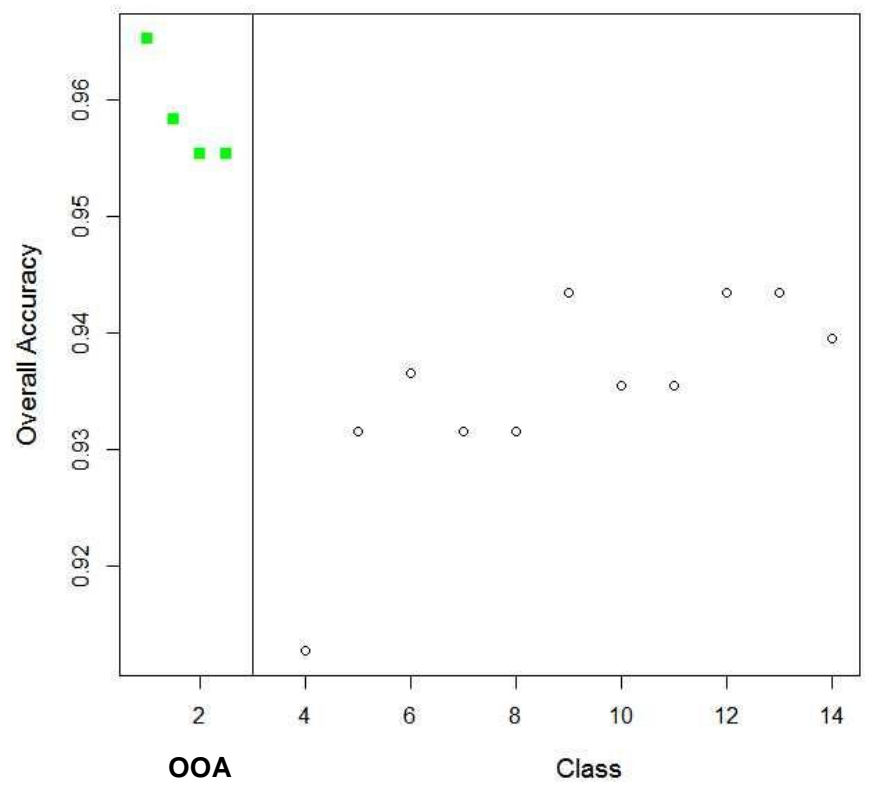

Figure 11: Overall accuracy of OOA and unsupervised classification results. OOA scale factors are plotted in green squares incrementally from 44-60. Class 4-14 indicate unsupervised classification levels as indicated by number. All equations and values may be referenced in Appendix 1.

Overall accuracy maximums were obtained at unsupervised classification levels of 9, 12 and 13 with a value of $94.3 \%$ while the maximum overall accuracy for OOA methods was found using a segmentation value of 44 and had an accuracy of $96.5 \%$. All OOA thresholds tested appear to outperform the best unsupervised classification as can be seen in Figures 10 and 11 . The statistical significance of these values can be seen in Figure 12.

Overall accuracy does not provide a complete picture of the accuracy of the methods, however. Because it is the arithmetic mean, there is no weight given to the variables and the number of non-landslide reference points outnumbers the number of landslides points by nearly a factor of 8 . This lead to data skewed toward proper nonlandslide identification. It is important to view each result to determine the strengths 
and weaknesses of each test and to fully understand the quality of the result. When examining the recall values of landslide identification, it appears that unsupervised classification with 4 classes outperformed the best OOA by $14 \%$. The downfall of this is that 4 class unsupervised classification had by far the highest false positive identification which resulted in it having the lowest overall accuracy and F-score. It is simply identifying a much higher percentage of the study area than the other test cases.

The most accurate way of examining the result is with the use of F-score. This provides a balanced view of the accuracy of how well each test detects both landslide and nonlandslide cases because it takes into account the number of samples of each dataset through the use of precision and recall. Overall accuracy performs an arithmetic mean of all data points and non-landslide cases outnumber the landslide detection results nearly by a factor of 8 . F-score for OOA methods outperformed all unsupervised classification methods in both landslide and non-landslide cases. The best F-score for landslide identification for OOA and Unsupervised methods were $84.3 \%$ and $77.9 \%$ respectively. This difference highlights the effect of the low precision values achieved by unsupervised classification. Precision values of $57 \%-71 \%$ for pixel based and $87 \%$ for OOA demonstrate the poor false positive removal of unsupervised classification.

By examining the confidence interval it is possible to quantify the importance of the distinction between overall accuracy and F-score in this study. Figure 12 shows error bars for overall accuracy and F-score for the best performing OOA and unsupervised methods. The confidence interval $(\boldsymbol{C l})$ is calculated from Foody (2009):

$$
C I=\text { estimate } \pm z_{\alpha / 2} *(S E)
$$

Where the estimate is either overall accuracy or F-score, $\boldsymbol{z}_{\boldsymbol{\alpha} / \mathbf{2}}$ is the z-score for a $95 \%$ level of significance $(\alpha=0.05)$ so $z_{\alpha / 2}=1.96$, and $\boldsymbol{S E}$ is the standard error of the estimate following the equation: 


$$
S E_{p_{1}-p_{0}}=\sqrt{\frac{p_{1}\left(1-p_{1}\right)}{n_{1}}+\frac{p_{0}\left(1-p_{0}\right)}{n_{0}}}
$$

For the standard error, $\boldsymbol{p}_{\mathbf{1}}$ is the proportion (OA or F-score value) being assessed and $\boldsymbol{p}_{\mathbf{0}}$ is the proportion being compared to (the best result of the alternative method). $\boldsymbol{n}_{\boldsymbol{i}}$ indicates the total number of samples (validation points) and is 1009 for all calculations in this study.

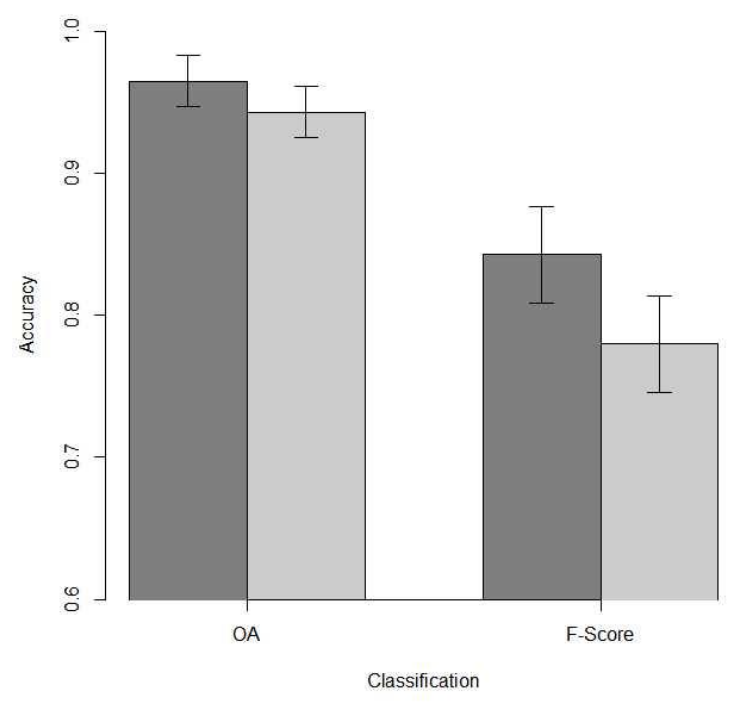

Figure 12: Confidence intervals of the top two performers in OOA and unsupervised classification (OOA darker gray) for Overall Accuracy (OA) and F-score. Calculated with 95\% level of significance (Foody, 2009).

Calculation of the confidence interval for overall accuracy and F-score validates that overall accuracy is not representative of the performance of the two methods.

Significant overlap in the error bars indicates there is no statistical significance to the performance of the two methods. F-score, however, has negligible overlap indicating that OOA outperforms unsupervised classification despite error in the result.

The performance of these methods must also take into account the limitations presented by the image, DEM, and validation dataset. Although numerical quantification is not available on the impact of these individually, it is important to note 
their existence. Many of these limitations are the result of poor data quality due to the remoteness of this region.

The high resolution ortho-photo is the best data available for this region, but it is not without its shortcomings. Shadowed areas are not correctable, and three landslides were located in these areas rendering them invisible to both unsupervised and OOA methods. These missed landslides can be seen in Figure 8. LiDAR imagery may have helped in the identification of landslides cloaked by these shadows (Van Den Eeckhaut et al, 2007).

Secondly, the low resolution of the DEM in comparison to the reference image limited the effectiveness of values derived from it. A flow accumulation layer proved unreliable in determining drainage paths and was therefore not used in the elimination of false positives in OOA. Had a higher quality DEM been available it may have been possible to reduce the number of false positives even more.

These drainage channels present another issue of importance. Without a proper field based map, it is often impossible to determine from the image alone where a landslide terminates and a drainage channel begins. An accurate measure of the full extent of each landslide would have added greatly to the assessment of the accuracy of these two methods.

Finally, because of the limitations of the dataset, a truly automated approach was not possible. There has been human influence despite all efforts being taken to process the data objectively. This is simply a challenge of assessing a historical dataset which does not allow field work to complement existing data. The combined effects of these limitations may be assessed in comparison to other similar research done on this topic (Chang et al 2012; Oruc et al. (2011); Whiteside et al.; Yan et al. 2006). Although methods varied in these papers, overall accuracy results for pixel based versus object oriented approaches varied from $38 \%$ improvement to $4 \%$ improvement. Results were most similar to those in Chang et al. (2012) which also examined pixel versus object 
based mapping of landslides. The result of this study fit well within this range validating the effectiveness of the methods performed.

\section{Conclusions}

The mapping of landslides at San Juan La Laguna, Guatemala by unsupervised classification and Object Oriented Analysis proved moderately successful. Despite data limitations, overall accuracies of $96.5 \%$ and $94.3 \%$ were obtained using OOA and unsupervised classification methods respectively. After confidence interval testing, it was determined that this improvement was not statistically relevant. The overall accuracy is skewed toward non-landslide identification, and does not accurately represent the effectiveness of the methods. The highest F-score values for landslide identification were $84.3 \%$ and $77.9 \%$ for OOA and unsupervised classification respectively. This difference of $6.4 \%$ more accurately represents the success of OOA methods than overall accuracy. By weighting the low precision values of unsupervised classification equally with the high recall values achieved with both methods, a more balanced result is achieved than overall accuracy provides. This was found to have negligible overlap of error bars, and is therefore considered statistically significant.

The low precision values for landslide identification using unsupervised classification highlight the greatest weakness of this method. False positive removal is limited to pixel values rather than object characteristics making the removal much more challenging when little is known of the study area and landslide characteristics. OOA has some limitations as well. Segmentation results showed that a plateau objective function may not properly identify the ideal scale factor for multiresolution segmentation. The best result falling below the plateau indicates that further assessment of the procedural accuracy and contextual application of this approach should be conducted for varying terrain, land cover, and land use scenarios. Additionally, brightness thresholding can be affected by rooftops, roads, and parking lots whose high brightness features influenced K-means clustering significantly. The 
automated methods described in Martha (2011) proved only partially successful in this study because the characteristics of the developed region dominated the image classification thresholds. Large urban areas create a unique challenge in the processing of remotely sensed data and should be analyzed cautiously on a case by case basis. This is especially true with images of a high enough resolution that individual buildings and small structures may be assigned image objects of their own. A potential solution would be the use of multiple segmentation values optimized for the varying dominant regions of the image.

The greatest limitation for both methods is the inability to confirm the extent of landslides with no ground truth available. A completed landslide inventory of this region would require significant speculation of landslide extent, especially where landslides intersect drainage channels which are spectrally and texturally similar. Despite these setbacks, OOA was able to identify the majority of the landslides to some extent with few false positive cases using only a high resolution orthophoto and DEM.

\section{Acknowledgements}

This material is based upon work partially supported by the National Science Foundation (NSF) Partnership for International Research and Education (PIRE) Grant N. 0530109. Special thanks to my committee: Dr. John Gierke and Dr. Mike Falkowski. To Dr. Tapas R. Martha for support in understanding and implementing his methods for OOA. To Patrice Coban for her Peace Corps service and collaboration with INSIVUMEH which aided greatly in the completion of this research. To Rudiger Wolf, Aaron Poznanovic, and Steven Gromatka for assistance and support. And especially to my advisor Dr. Thomas Oommen for his incredible patience, support and knowledge throughout the entire process. 


\section{References}

Barlow, J., Franklin, S., and Martin, Y. "High spatial resolution satellite imagery, DEM derivatives, and image segmentation for the detection of mass wasting processes", Photogramm. Eng. Rem. S., 72(6), 687-692, 2006. Web. Nov. 2012.

Borghuis, A. M., K. Chang, and H. Y. Lee. "Comparison between Automated and Manual Mapping of Typhoon-triggered Landslides from SPOT-5 Imagery."International Journal of Remote Sensing. Taylor \& Francis, 28 Dec. 2007. Web. Nov. 2012.

Chang, Kuan-Tsung, Jun-King Liu, and Chu-I Wang. "An Object-oriented Analysis for Characterizing the Rainfall-induced Shallow Landslide." Journal of Marine Science and Technology 20.6 (2012): 647-56. Web. Dec. 2012

eCognition, 2012. eCognition Developer 8. Version 8.8. Trimble.

Erdas Imagine,2011. Version 11.0.2

Espindola, G. M., G. Camara, I. A. Reis, L. S. Bins, and A. M. Monteiro. "Parameter Selection for Region-Growing Imagef Segmentation Algorithms Using Spatial Autocorrelation." International Journal of Remote Sensing (2006): CiteSeer. Web. Oct. 2012.

ESRI (Environmental Systems Resource Institute). 2010. ArcMap 10.0. ESRI, Redlands, California.

Foody, Giles M. "Classification accuracy comparison: hypothesis tests and the use of confidence intervals in evaluations of difference, equivalence and noninferiority." Remote Sensing of Environment 113.8 (2009): 1658-1663.

IBM Corp. Released 2010. IBM SPSS Statistics for Windows, Version 19.0. Armonk, NY: IBM Corp.

Lahousse, T., K. T. Chang, and Y. H. Lin. "Landslide Mapping with Multi-Scale ObjectBased Image Analysis - A Case Study in the Baichi Watershed, Taiwan." Natural Hazards and Earth Systems Sciences 11.2715 (2011): Nat. Hazards Earth Syst. Sci. Copernicus Publications, 10 Oct. 2011. Web. Oct. 2012. 
Lu, Ping; Stumpf, A.; Kerle, N.; Casagli, N., "Object-Oriented Change Detection for Landslide Rapid Mapping," Geoscience and Remote Sensing Letters, IEEE , vol.8, no.4, pp.701,705, July 2011

Luna, Byron Quan. "Assessment and modelling of two lahars caused by "Hurricane Stan" at Atitlan, Guatemala, October 2005," Masteroppgave, University of Oslo, 2007. Web. Feb. 2013.

Martha, Tapas R. Detection of Landslides by Object Oriented Image Analysis. Diss. University of Twente, 2011. Enschede: ITC, 2011. Print. Oct. 2011

Martha, Tapas R., Norman Kerle, Victor Jetten, Cees J. Van Westen, and K. Vinod Kumar. "Characterising Spectral, Spatial and Morphometric Properties of Landslides for Semi-Automatic Detection Using Object-Oriented Methods." Geomophology 116 (2010): 24-36. Elsevier. Web. Feb. 2012.

Martha, Tapas R., Norman Kerle, Cees J. Van Westen, Victor Jetten, and K. Vinod Kumar. "Object-Oriented Analysis of Multi-Temporal Panchromatic Images for Creation of Historical Landslide Inventories." ISPRS Journal of Photogrammetry and Remote Sensing 67 (2012): 105-19. Elsevier. Web. Sept. 2012.

Newhall, C. G., C. K. Paull, J. P. Bradbury, A. Higuera-Gundy, L. J. Poppe, S. Self, N. Bonar Sharpless, and J. Ziagos. "Recent Geologic History of Lake Atitlan, A Caldera Lake in Western Guatemala." Journal of Volcanology and Geothermal Research 33 (1987): 81-107. Web. Jan. 2013.

Oommen, T., Baise, L., and Vogel, R. "Validation and Application of Empirical Liquefaction Models." J. Geotech. Geoenviron. Eng., (2010): 136(12), 16181633. Web. Nov. 2012

O'Neil-Dunne, Jarlath. "Letters from the SAL.": ECognition. University of Vermont, 13 Apr. 2010. Web. Feb. 2013.

Oruc, M., A. M. Marangoz, and G. Buyuksalih. Comparison of Pixel-based and Object Oriented Classification Approaches Using Landsat-7 ETM Spectral Bands. Thesis. Konguldak Karaelmas University. 2011. Web. Jan 2013.

R Development Core Team (2008). R: A language and environment for statistical computing. R Foundation for Statistical Computing,Vienna, Austria. ISBN 3900051-07-0, URL http://www.R-project.org. 
Ruedi, Boesch, and Wang Zuyuan. "Segmentation Optimization for Aerial Images with Spatial Constraints." Photogrammetry, Remote Sensining and Spatial Information Sciences 37 (2008): n. pag. International Society of Photogrammetry and Remote Sensing. Sept. 2012. Web. Jan. 2013

Sandric, I., B. Mihai, Z. Chitu, A. Gutu, and I. Savulescu. "Object-Oriented Methods for Landslide Detection Using High Resolution Imagery, Morphometric Properties and Meteorological Data." ISPRS 38 (2010): n. pag. Web. Nov. 2012.

Schwarz, Gideon. "Estimating the Dimension of a Model." The Annals of Statistics 6.2 (1978): 461-64. JSTOR. Web. Nov. 2012.

Stumpf, Andre and Norman Kerle "Combining Random Forests and object-oriented analysis for landslide mapping from very high resolution imagery", Procedia Environmental Sciences, Volume 3 (2011): 123-129. Web. Oct. 2012

Van Den Eeckhaut, Miet, Norman Kerle, and Javier Hervas. Mapping Vegetated Landslides Using LiDAR Derivatives and Object-oriented Analysis. Thesis. University of Twente. 2007. Print. Oct. 2012

Whiteside, T., and W. Ahmad. "A Comparison of Object-oriented and Pixel-based Classification Methods for Mapping Land Cover in Northern Australia." Spatial Intelligence, Innovation and Praxis (2005): n. pag. ECognition. Web. Feb. 2013.

Yan, Gao, J. F. Mas, B. H. P Maathuis, Zhang Xiangmin, and P. M. Van Dijk. "Comparison of Pixel-based and Object-oriented Image Classification Approaches - A Case Study in a Coal Fire Area, Wuda, Inner Mongolia, China." International Journal of Remote Sensing 27.18 (2006): 4039-055. Print. Dec. 2012. 


\section{APPENDIX I: Accuracy Assessment Data}

$$
\begin{gathered}
\text { Precision }=P=\frac{T P}{T P+F P} \\
\text { Recall }=R=\frac{T P}{T P+F N} \\
F \text { score }=F_{\beta}=\frac{\left(1+\beta^{2}\right)(P * R)}{\beta * P+R} \\
\text { Overall Accuracy }=(T P+T N) /(T P+T N+F P+F N)
\end{gathered}
$$

Where TP is the sum of all landslide instances identified correctly, TN is the sum of all nonlandslide instances identified correctly, FP is the sum of all non-landslides identified as landslides, FN is the sum of all landslides identified as non-landslides, and $\beta$ is the measure of the importance of precision to recall ( $\beta=1$ for this study indicating equal importance) (Oommen 2010).

\begin{tabular}{|l|r|r|r|r|r|r|r|r|}
\hline Name & Class & $\begin{array}{l}\text { Precision } \\
\text { Landslide }\end{array}$ & $\begin{array}{l}\text { Precision } \\
\text { Non-LS }\end{array}$ & $\begin{array}{l}\text { Recall } \\
\text { Landslide }\end{array}$ & $\begin{array}{l}\text { Recall } \\
\text { Non-LS }\end{array}$ & $\begin{array}{l}\text { Overall } \\
\text { Accuracy }\end{array}$ & $\begin{array}{l}\text { F score } \\
\text { Landslide }\end{array}$ & $\begin{array}{l}\text { F score } \\
\text { Non-LS }\end{array}$ \\
\hline object44 & 1 & 0.8704 & 0.9767 & 0.8174 & 0.9843 & 0.9653 & 0.8430 & 0.9805 \\
\hline object60 & 2 & 0.8710 & 0.9629 & 0.7043 & 0.9866 & 0.9544 & 0.7788 & 0.9746 \\
\hline unsup4 & 4 & 0.5699 & 0.9939 & 0.9565 & 0.9072 & 0.9128 & 0.7143 & 0.9485 \\
\hline unsup5 & 5 & 0.6337 & 0.9928 & 0.9478 & 0.9295 & 0.9316 & 0.7596 & 0.9601 \\
\hline unsup6 & 6 & 0.6604 & 0.9882 & 0.9130 & 0.9396 & 0.9366 & 0.7664 & 0.9633 \\
\hline unsup7 & 7 & 0.6337 & 0.9928 & 0.9478 & 0.9295 & 0.9316 & 0.7596 & 0.9601 \\
\hline unsup8 & 8 & 0.6337 & 0.9928 & 0.9478 & 0.9295 & 0.9316 & 0.7596 & 0.9601 \\
\hline unsup9 & 9 & 0.7014 & 0.9838 & 0.8783 & 0.9519 & 0.9435 & 0.7799 & 0.9676 \\
\hline unsup10 & 10 & 0.6563 & 0.9882 & 0.9130 & 0.9385 & 0.9356 & 0.7636 & 0.9627 \\
\hline unsup11 & 11 & 0.6563 & 0.9882 & 0.9130 & 0.9385 & 0.9356 & 0.7636 & 0.9627 \\
\hline unsup12 & 12 & 0.7014 & 0.9838 & 0.8783 & 0.9519 & 0.9435 & 0.7799 & 0.9676 \\
\hline unsup13 & 13 & 0.7014 & 0.9838 & 0.8783 & 0.9519 & 0.9435 & 0.7799 & 0.9676 \\
\hline unsup14 & 14 & 0.7143 & 0.9717 & 0.7826 & 0.9597 & 0.9395 & 0.7469 & 0.9657 \\
\hline
\end{tabular}

Table 1: Complete table of all precision, recall, $f$ score, and overall accuracy data from analysis

\section{References}

Oommen, T., Baise, L., and Vogel, R. (2010). "Validation and Application of Empirical Liquefaction Models." J. Geotech. Geoenviron. Eng., 136(12), 1618-1633. 


\section{APPENDIX 2: Landslide Inventory and Validation}

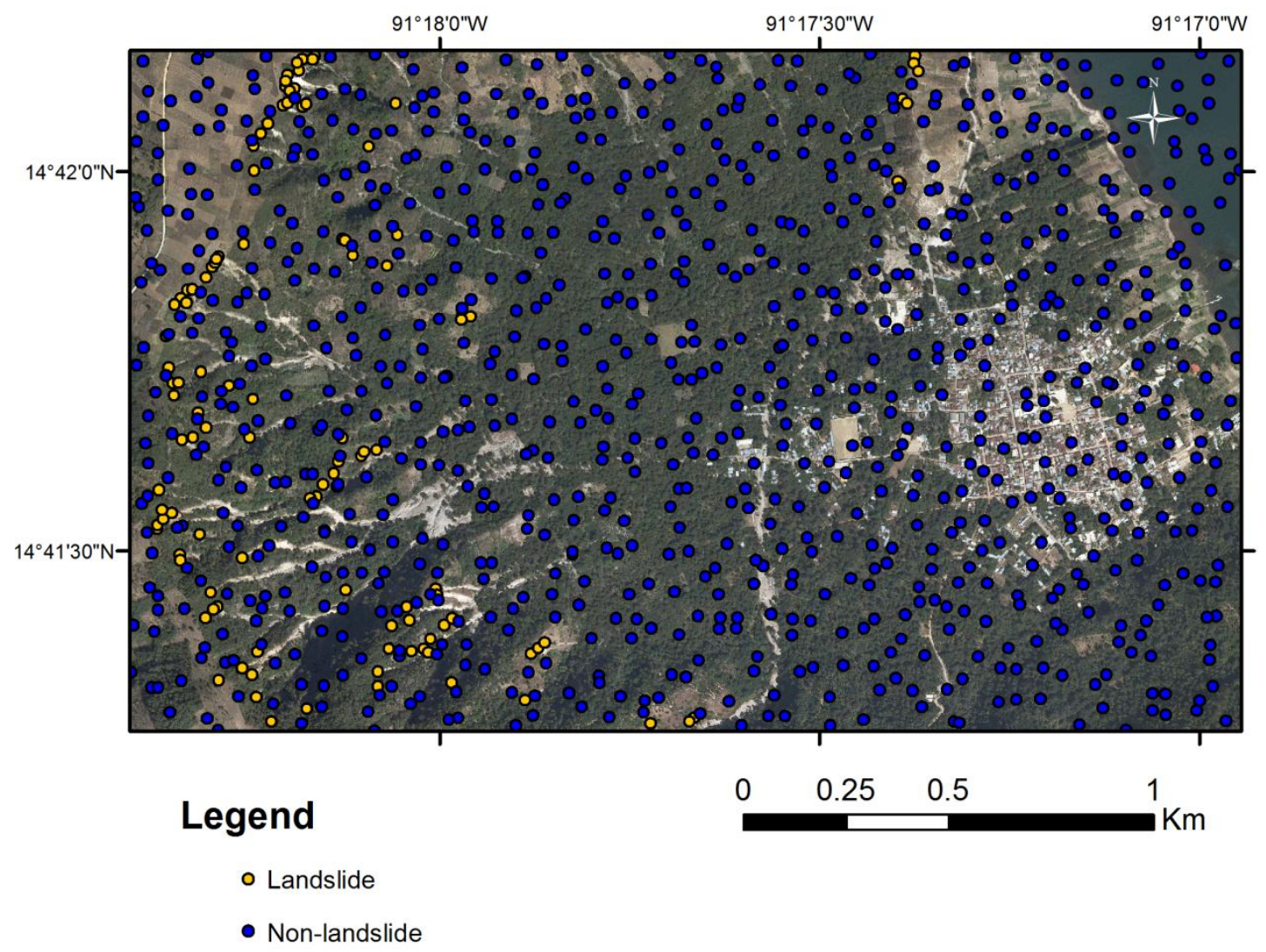

Figure 1: Study area with validation data point overlay. Yellow landslide points were identified by INSIVUMEH scientists shortly after the event. Blue non-landslide points were selected randomly in ArcGIS. There are 115 landslide and 894 non-landslide cases. 